\title{
Quantum theory of optomechanical cooling
}

\author{
Florian Marquardt (1), A. A. Clerk (2), and S. M. Girvin (3)
}

July 17, 2021

(1) Sektion Physik, Ludwig-Maximilians-Universität München, Theresienstr. 37, D-80333 München, Germany; (2) Department of Physics, McGill University, 3600 rue University, Montreal, QC Canada H3A 2T8; (3) Department of Physics, Yale University, PO Box 208120, New Haven, CT 06520-8120

\begin{abstract}
We review the quantum theory of cooling of a mechanical oscillator subject to the radiation pressure force due to light circulating inside a driven optical cavity. Such optomechanical setups have been used recently in a series of experiments by various groups to cool mechanical oscillators (such as cantilevers) by factors reaching $10^{5}$, and they may soon go to the ground state of mechanical motion. We emphasize the importance of the sideband-resolved regime for ground state cooling, where the cavity ring-down rate is smaller than the mechanical frequency. Moreover, we illustrate the strong coupling regime, where the cooling rate exceeds the cavity ring-down rate and where the driven cavity resonance and the mechanical oscillation hybridize. Keywords: cavity QED, optomechanics, micromechanics, sideband cooling, radiation pressure
\end{abstract}

\section{Introduction}

The interaction of light with matter has been at the heart of the development of quantum mechanics since its inception. As for the mechanical effects of light, these become most pronounced in a setup where the light intensity is resonantly enhanced (i.e. an optical cavity) and where photons transfer maximum momentum to a mechanical object, e.g. by being reflected multiple times from a movable mirror attached to a cantilever. The study of radiation pressure effects on a movable mirror was pioneered in seminal papers by Braginsky [1, 2]. Strong changes of the mechanical properties of the mirror were observed later in an experiment by the Walther group [3], where a macroscopic mirror was found to exhibit two stable equilibrium positions under the action of the cavity's radiation field. The most recent series of activity in this field started with experiments observing optomechanical cooling first using feedback [4, 5] and later [6, 7, 8, 9, 10, 11, 12, using the intrinsic effect discussed in the following. In addition, we note the self-induced optomechanical oscillations [13] that 


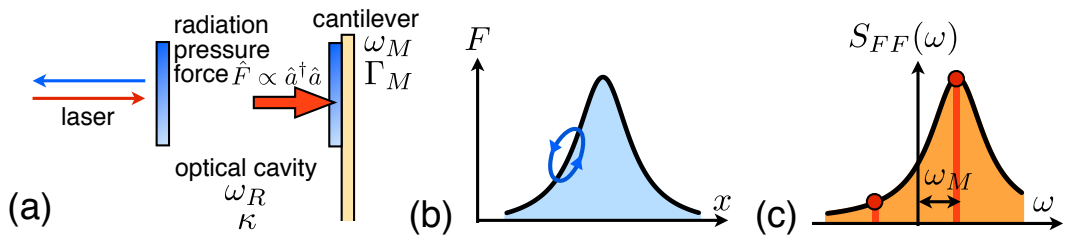

Figure 1: (a) The standard optomechanical setup treated in the text: A driven optical cavity with a movable mirror. (b) Moving the mirror in a cycle can result in work extracted by the light-field, due to the finite cavity ring-down rate. (c) Radiation pressure force noise spectrum.

have been observed in radiation-pressure driven microtoroidal optical resonators [14, 15] and other setups [16, 17. For a recent review see [18]. The study of these systems has been made even more fruitful by the realization that the same (or essentially similar) physics may be observed in systems ranging from driven LC circuits coupled to cantilevers [19] over superconducting single electron transistors and microwave cavities coupled to nanobeams $20,21,22,23,24,25,26$, to clouds of cold atoms in an optical lattice, whose oscillations couple to the light field [27, 28. Cooling to the ground-state may open the door to various quantum effects in these systems, including "cat" states [29], entanglement [30, 31] and Fock state detection [12.

All the intrinsic optomechanical cooling experiments are based on the fact that the radiation field introduces extra damping for the cantilever. In such a classical picture, the effective temperature of the single mechanical mode of interest is related to the bath temperature $T$ by $T_{\text {eff }} / T=\Gamma_{M} /\left(\Gamma_{\text {opt }}+\Gamma_{M}\right)$, where $\Gamma_{M}$ and $\Gamma_{\mathrm{opt}}$ are the intrinsic mechanical damping rate and the optomechanical cooling rate, respectively. Thus there is no limit to cooling in this regime, provided the laser power (and thus the cooling rate $\Gamma_{\mathrm{opt}}$ ) can be increased without any deleterious effects such as unwanted heating by absorption, and provided the cooling rate remains sufficiently smaller than the mechanical frequency and the cavity ring-down rate. However, at sufficiently low temperatures, the unavoidable photon shot noise inside the cavity counteracts cooling. To study the resulting quantum limits to cooling, a fully quantum-mechanical theory is called for, which we provided in Ref. [32, based on the general quantum noise approach. Independently, a derivation emphasizing the analogy to ion sideband cooling was developed in Ref. 33. In the present paper, we will review and illustrate our theory. We start by outlining the basic classical picture, then present the quantum noise approach that provides a transparent and straightforward way to derive cooling rates and quantum limits for the phonon number. Finally, we illustrate the strong coupling regime that was first predicted in our Ref. [32]. 


\section{Basic classical picture}

In this section, we briefly review the basic classical description of optomechanical cooling.

The main ingredient for optomechanical cooling is the appearance of extra damping, ideally without extra fluctuations. This damping is introduced because the light-induced force reacts with a finite delay time. In the case of radiation pressure, this comes about due to the ring-down time $\kappa^{-1}$ of the cavity. On the other hand, bolometric (i.e. photothermal) forces are produced when a bimorph cantilever absorbs some of the radiation circulating inside the cavity. When bolometric forces dominate, it is the finite time of thermal conductance that sets the time-lag between the impinging radiation intensity and the resulting change in the cantilever temperature, which is proportional to the force.

The physical picture behind damping is simplest when the time-lag is small compared to the oscillation period of the cantilever. Then both radiation pressure and bolometric forces give rise to the same physics, modulo the appearance of a different time-scale in the two cases. To describe this physics, we first fix our coordinate system: Increasing the displacement $x$ of the cantilever means elongating the cavity, and thus the optical resonance frequency (of the mode of interest) decreases. Let us consider the cantilever being placed at some location to the left of the resonance (Fig. 1). This means that the optical mode frequency is still higher than the frequency of the incoming laser radiation, which is therefore red-detuned with respect to the optical resonance. Now imagine moving the cantilever in a small cycle. As it moves towards the resonance with a finite speed, the light-induced force does work on the cantilever. However, due to the time-lag it remains smaller than it would be in the case of infinitely slow (adiabatic) motion. Conversely, as the cantilever moves back again in the second half of the cycle, the force extracts energy and it is larger than for adiabatic motion. In total, the work done during such a cycle by the light-induced force is negative, i.e. mechanical energy is extracted from the cantilever (Fig. 1p). This kind of physics may be modeled by writing down a simple relaxation-type equation for the force, which tries to reach its proper $x$-dependent value $\mathcal{F}(x)$ with some time-lag:

$$
\dot{F}(t)=(\mathcal{F}(x(t))-F(t)) / \tau .
$$

The cantilever is a damped harmonic oscillator driven by the light-induced force:

$$
m \ddot{x}=-m \omega_{M}^{2}\left(x-x_{0}\right)-m \Gamma_{M} \dot{x}+F,
$$

where $x_{0}$ is the mechanical equilibrium position, $m$ the cantilever mass, $\omega_{M}$ its mechnical frequency, and $\Gamma_{M}$ the intrinsic damping rate. Linearizing equation (1) with respect to small displacements from the mechanical equilibrium position $\bar{x}$ and inserting it into the equation of motion of the cantilever then yields the extra damping force. In Fourier space, where $x(t)=\int x[\omega] e^{-i \omega t} d \omega / 2 \pi$, we find the following linearized equation of motion (at $\omega \neq 0$ ): 


$$
-\omega^{2} m x[\omega]=-m \omega_{M}^{2} x[\omega]+i m \omega \Gamma_{M} x[\omega]+\mathcal{F}^{\prime}(\bar{x}) x[\omega] /(1-i \omega \tau)
$$

Comparing the last two terms (the intrinsic damping with the imaginary part of the optomechanical term), we find that the optomechanical damping rate is given by

$$
\Gamma_{\mathrm{opt}}=\frac{\mathcal{F}^{\prime}(\bar{x})}{m \omega_{M}} \frac{\omega_{M} \tau}{1+\left(\omega_{M} \tau\right)^{2}},
$$

for the simple ansatz of Eq. (1). According to this analysis, one would expect the maximum effect to occur when $\omega_{M} \tau=1$, i.e. when the time-delay matches the period of the cantilever motion. As we will see further below, this conclusion is not upheld by the full quantum-mechanical analysis for the case of radiation pressure.

Equation (1) and the subsequent analysis holds exactly for the bolometric force. In that case, $\tau$ is the finite time of thermal conductance and $\mathcal{F}(x)=$ $\mathcal{F}_{\max } I(x) / I_{\max }$ is the displacement-dependent bolometric force, where $I(x)=$ $I_{\max } /\left(1+(2 \Delta(x) / \kappa)^{2}\right)$ is the intensity profile and $\Delta(x)=x \omega_{R} / L$ is the positiondependent detuning between incoming laser radiation and optical resonance at $x=0$. For the radiation-pressure force, we can use the present analysis only in the regime of $\kappa \gg \omega_{M}$, and only if we allow for a position dependent relaxation rate $1 / \tau$ (see [32]).

In both cases, however, the shape of $\Gamma_{\text {opt }}$ as a function of cantilever position $\bar{x}$ is determined by the slope of the intensity profile, i.e. in particular by the sign of $\mathcal{F}^{\prime}$. To the left of the resonance, where $\mathcal{F}^{\prime}>0$, we indeed obtain extra damping: $\Gamma_{\mathrm{opt}}>0$. As long as there are no extra fluctuations introduced by the light-induced force (i.e. if we may disregard shot noise), the effective temperature of the mechanical degree of freedom is therefore reduced according to the ratio of intrinsic and optomechanical damping rates:

$$
T_{\text {eff }}=T \frac{\Gamma_{M}}{\Gamma_{M}+\Gamma_{\mathrm{opt}}} .
$$

This can be obtained, for example, by solving the Langevin equation that includes the thermal fluctuations of the mechanical heat bath (whose strength is set by $\Gamma_{M}$ according to the fluctuation-dissipation theorem). Then the effective temperature may be defined according to the equipartition theorem: $m \tilde{\omega}_{M}^{2}\left\langle(x-\bar{x})^{2}\right\rangle=k_{B} T_{\text {eff }}$, where $\tilde{\omega}_{M}$ contains the frequency renormalization due to the real part of the optomechanical term in Eq. (3).

\section{Quantum noise approach}

In the quantum regime, we have to take into account the shot noise that tends to heat the cantilever and enforces a finite quantum limit for the cantilever phonon number. The quantum picture can also be understood as Raman scattering: 
Incoming photons, red-detuned with respect to the optical resonance, absorb a phonon from the cantilever, thereby cooling it. However, there is also a finite probability for phonon emission, and thus heating. The purpose of a quantum theory is to discuss the balance of these effects.

The idea behind the quantum noise approach to quantum-dissipative systems is to describe the environment fully by the correlator of the fluctuating force that couples to the quantum system of interest. If the coupling is weak enough, knowledge of the correlator is sufficient to fully describe the influence of the environment. In our case, this means looking at the spectrum of the radiation pressure force fluctuations, which are produced by the shot noise of photons inside the driven optical cavity mode, i.e. a nonequilibrium environment. In other applications, we might be dealing with the electrical field fluctuations produced, e.g., by a driven electronic circuit (superconducting single electron transistor, quantum point contact, LC circuit) capacitively coupled to some nanobeam. The general formulas remain the same for all of these cases, and only the noise spectrum changes.

The Fourier transform of the force correlator defines the spectral noise density:

$$
S_{F F}(\omega)=\int d t e^{i \omega t}\langle\hat{F}(t) \hat{F}(0)\rangle .
$$

The noise spectrum $S_{F F}$ is real-valued and non-negative. However, in contrast to the classical case, it is asymmetric in frequency, since $\hat{F}(t)$ and $\hat{F}(0)$ do not commute. This asymmetry has an important physical meaning: Contributions at positive frequencies indicate the possibility of the environment to absorb energy, while those at negative frequencies imply its ability to release energy (to the cantilever). All the optomechanical effects can be described in terms of $S_{F F}$, as long as the coupling is weak.

The optomechanical damping rate is given by the difference of noise spectra at positive and negative frequencies,

$$
\Gamma_{\mathrm{opt}}=\frac{x_{\mathrm{ZPF}}^{2}}{\hbar^{2}}\left[S_{F F}\left(\omega_{M}\right)-S_{F F}\left(-\omega_{M}\right)\right] .
$$

This formula is obtained by applying Fermi's Golden Rule to derive the transition rates arising from the coupling of the cantilever to the light field, i.e. from the term $\hat{H}_{\mathrm{int}}=-\hat{F} \hat{x}$ in the Hamiltonian. These are

$$
\Gamma_{\downarrow}^{\mathrm{opt}}=\frac{x_{\mathrm{ZPF}}^{2}}{\hbar^{2}} S_{F F}\left(\omega_{M}\right) \quad, \quad \Gamma_{\uparrow}^{\mathrm{opt}}=\frac{x_{\mathrm{ZPF}}^{2}}{\hbar^{2}} S_{F F}\left(-\omega_{M}\right) .
$$

These rates enter the complete master equation for the density matrix $\hat{\rho}$ of the cantilever in the presence of the equilibrium heat bath (that would lead to a thermal population $\bar{n}_{\mathrm{th}}$ ) and the radiation field:

$$
\dot{\hat{\rho}}=\left[\left(\Gamma_{\downarrow}^{\mathrm{opt}}+\Gamma_{M}\left(\bar{n}_{\mathrm{th}}+1\right)\right) \mathcal{D}[\hat{a}]+\left(\Gamma_{\uparrow}^{\mathrm{opt}}+\Gamma_{M} \bar{n}_{\mathrm{th}}\right) \mathcal{D}\left[\hat{a}^{\dagger}\right]\right] \hat{\rho}
$$



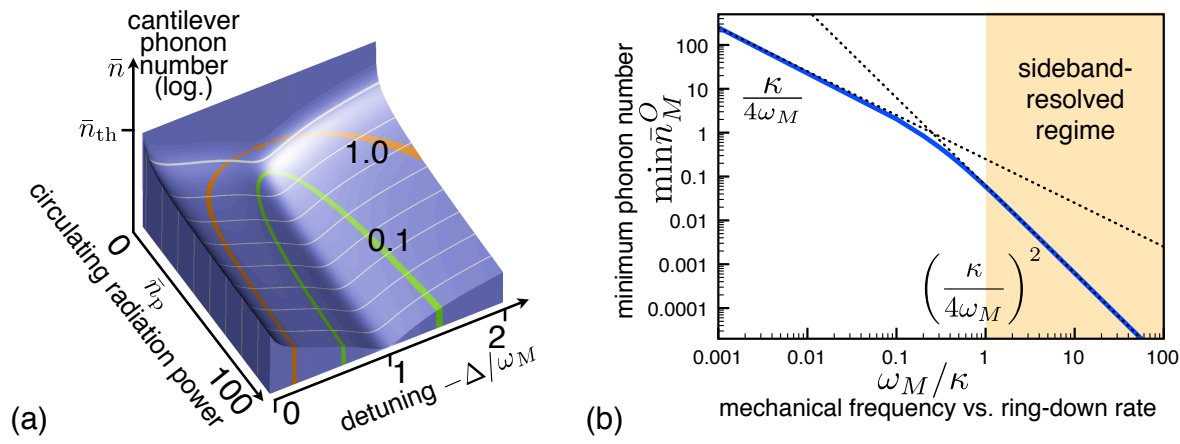

Figure 2: (a) Cantilever phonon number as a function of circulating radiation power inside the cavity and as a function of detuning between laser and optical resonance. The mean phonon number in steady state is plotted on a logarithmic scale. Contour lines indicate $\bar{n}=1$ and $\bar{n}=0.1$. For this plot, the following parameters have been used: $\omega_{M} / \kappa=0.3, \bar{n}_{\mathrm{th}}=10^{3}, Q=\omega_{M} / \Gamma_{M}=10^{6}$, and $\left(\omega_{R} / \omega_{M}\right)\left(x_{\mathrm{ZPF}} / L\right) \approx 0.012$. (b) The minimum phonon number as a function of the ratio between the mechanical frequency $\omega_{M}$ and the optical cavity's ringdown rate $\kappa$, according to Eq. (15). Ground-state cooling is possible in the regime $\omega_{M} \gg \kappa$, i.e. the "good cavity" or "resolved sideband" limit.

Here the equation has been written in the interaction picture (disregarding the oscillations at $\tilde{\omega}_{M}$ ), and

$$
\mathcal{D}[\hat{A}] \hat{\rho}=\frac{1}{2}\left(2 \hat{A} \hat{\rho} \hat{A}^{\dagger}-\hat{A}^{\dagger} \hat{A} \hat{\rho}-\hat{\rho} \hat{A}^{\dagger} \hat{A}\right)
$$

is the standard Lindblad operator for downward $(\hat{A}=\hat{a})$ or upward $\left(\hat{A}=\hat{a}^{\dagger}\right)$ transitions in the oscillator. Restricting ourselves to the populations $\rho_{n n}$, we obtain the equation for the phonon number $\bar{n}=\langle\hat{n}\rangle=\sum_{n} n \rho_{n n}$ :

$$
\dot{\bar{n}}=\Gamma_{M} \bar{n}_{\mathrm{th}}+\Gamma_{\uparrow}^{\mathrm{opt}}-\left(\Gamma_{M}+\Gamma_{\mathrm{opt}}\right) \bar{n},
$$

which yields the steady-state phonon number in the presence of optomechanical cooling:

$$
\bar{n}_{M}=\frac{\Gamma_{M} \bar{n}_{\mathrm{th}}+\Gamma_{\mathrm{opt}} \bar{n}_{M}^{O}}{\Gamma_{M}+\Gamma_{\mathrm{opt}}} .
$$

This is the weighted average of the thermal and the optomechanical phonon numbers. It represents the correct generalization of the classical formula for the effective temperature, Eq. (5). Here

$$
\bar{n}_{M}^{O}=\frac{\Gamma_{\uparrow}^{\mathrm{opt}}}{\Gamma_{\mathrm{opt}}}=\frac{1}{\Gamma_{\downarrow}^{\mathrm{opt}} / \Gamma_{\uparrow}^{\mathrm{opt}}-1}=\left[\frac{S_{F F}\left(\omega_{M}\right)}{S_{F F}\left(-\omega_{M}\right)}-1\right]^{-1}
$$


is the minimal phonon number reachable by optomechanical cooling. This quantum limit is reached when $\Gamma_{\text {opt }} \gg \Gamma_{M}$. Then, the cooling effect due to extra damping is balanced by the shot noise in the cavity, which leads to heating.

The radiation pressure force is proportional to the photon number: $\hat{F}=$ $\left(\hbar \omega_{R} / L\right) \hat{a}^{\dagger} \hat{a}$. A brief calculation for the photon number correlator inside a driven cavity 32 yields its spectrum in the form of a Lorentzian that is shifted by the detuning $\Delta=\omega_{L}-\omega_{R}$ between laser and optical resonance frequency $\omega_{R}$ :

$$
S_{F F}(\omega)=\left(\frac{\hbar \omega_{R}}{L}\right)^{2} \bar{n}_{\mathrm{p}} \frac{\kappa}{(\omega+\Delta)^{2}+(\kappa / 2)^{2}},
$$

where $\bar{n}_{p}$ is the photon number circulating inside the cavity. A plot of the resulting steady-state phonon number is shown in Fig. 2 a.

Inserting this spectrum into Eqs. (7) and (13) yields the optomechanical cooling rate and the minimum phonon number as a function of detuning $\Delta$. The minimum of $\bar{n}_{M}^{O}$ is reached at a detuning $\Delta=-\sqrt{\omega_{M}^{2}+(\kappa / 2)^{2}}$, and it is (see Fig. 2p):

$$
\min \bar{n}_{M}^{O}=\frac{1}{2}\left(\sqrt{1+\left(\frac{\kappa}{2 \omega_{M}}\right)^{2}}-1\right) .
$$

For slow cantilevers, $\omega_{M} \ll \kappa$, we have $\min \bar{n}_{M}^{O}=\kappa /\left(4 \omega_{M}\right) \gg 1$. Ground-state cooling becomes possible for high-frequency cantilevers (and/or high-finesse cavities), when $\kappa \ll \omega_{M}$. Then, we find

$$
\min \bar{n}_{M}^{O} \approx\left(\frac{\kappa}{4 \omega_{M}}\right)^{2}
$$

As explained in Ref. 33, these two regimes can be brought directly into correspondence with the known regimes for laser-cooling of harmonically bound atoms, namely the Doppler limit for $\omega_{M} \ll \kappa$ and the resolved sideband regime for $\omega_{M} \gg \kappa$.

\section{$4 \quad$ Strong coupling effects}

Up to now, we have assumed that the coupling between light and mechanical degree of freedom is sufficiently weak to allow for a solution in terms of a master equation, employing the rates obtained from the quantum noise approach. However, as the coupling becomes stronger (e.g. by increasing the laser input power), $\Gamma_{\text {opt }}$ may reach the cavity decay rate $\kappa$. Then, the spectrum of force fluctuations is itself modified by the presence of the cantilever. It becomes necessary to solve for the coupled dynamics of the light field and the mechanical motion. This has been done in Ref. 32, by writing down the Heisenberg equations of motion for the cantilever and the optical mode, and solving them after linearization. Here we will only discuss the result. 


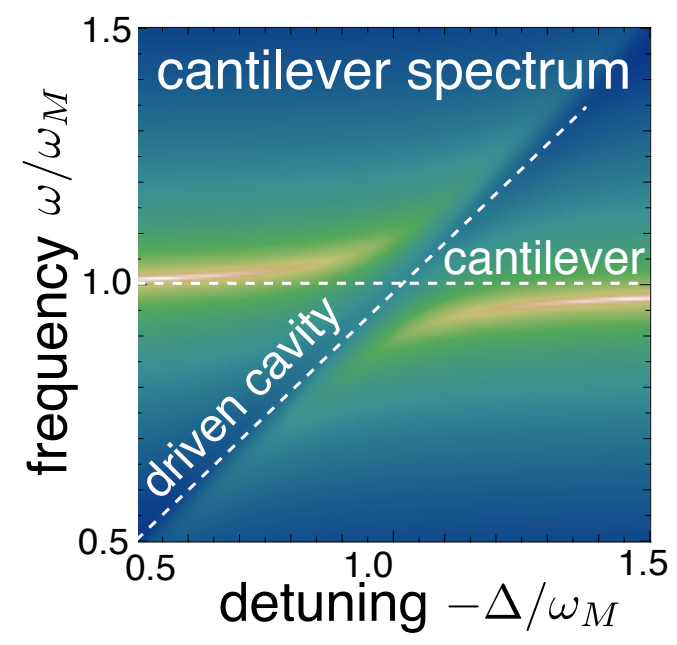

Figure 3: The spectrum $S_{c c}(-\omega)$ of the cantilever motion, as a function of detuning $\Delta$ and spectral frequency $\omega$, for fixed circulating power. For the plot shown here, the strong-coupling regime is reached, i.e. $\Gamma_{\text {opt }} / \kappa$ becomes larger than one near resonance. Consequently, the driven cavity resonance and the cantilever resonance hybridize, and an avoided crossing is observed. The parameters for this plot are: $\kappa / \omega_{M}=0.1, \Gamma_{M} / \omega_{M}=10^{-5},\left(\omega_{R} / \omega_{M}\right)\left(x_{\mathrm{ZPF}} / L\right)=0.01$, $\bar{n}_{p}=100$, and $\bar{n}_{\mathrm{th}}=10^{3}$.

To analyze these features, let us consider the spectrum of the cantilever motion,

$$
S_{c c}(\omega)=\int d t e^{i \omega t}\left\langle\hat{c}^{\dagger}(t) \hat{c}(0)\right\rangle,
$$

where $\hat{c}$ is the annihilation operator for the cantilever harmonic oscillator. At weak coupling, this spectrum displays a peak at the (renormalized) cantilever frequency, i.e. at $\omega=-\tilde{\omega}_{M}$ [the minus sign is a consequence of our choice of the definition, following [32]]. Its width (FWHM) is given by $\Gamma_{\mathrm{opt}}+\Gamma_{M}$, and its total weight $\int S_{c c}(\omega) d \omega / 2 \pi$ yields the phonon number $\bar{n}=\left\langle\hat{c}^{\dagger} \hat{c}\right\rangle$. As the laser power is increased, the width increases and the weight diminishes (in the cooling regime).

When $\Gamma_{\text {opt }} / \kappa$ is no longer much smaller than one, deviations from the weakcoupling results start to appear 32. The most dramatic effect is observed when $\Gamma_{\text {opt }} / \kappa>1 / 2$ : The peak splits into two (Fig. 3). As it were, in this strong-coupling regime one actually encounters the hybridization of two coupled harmonic oscillators, namely the cantilever and the driven optical mode (with an effective frequency set by the detuning). At resonance, i.e. for $\Delta=-\omega_{M}$, the splitting is set by $2 \alpha$, where the coupling frequency $\alpha$ is determined by the circulating laser power, the ratio of mechanical zero-point fluctuations to the 
cavity length, and by the optical resonance frequency:

$$
\alpha=\omega_{R} \sqrt{\bar{n}_{p}} \frac{x_{\mathrm{ZPF}}}{L} .
$$

\section{Outlook}

During the past year, several new ideas have been introduced into the field of optomechanical cooling. For example, placing a movable membrane in the middle of a standard optical cavity [12] can lead to orders of magnitude better performance, as it separates the mechanical from the optical elements. Such a setup has been used to cool from $300 K$ down to $7 m K$, and it may ultimately by employed for Fock state detection of mechanical vibrations [12. Even nanomechanical objects (such as nanowires) might be placed inside the standing light wave 34 and cooled by scattering. Doppler cooling of Bragg mirrors may provide another promising approach [35. Furthermore, sideband-resolved cooling with $\omega_{M} / \kappa \sim 20$ has been demonstrated recently [36], paving the way for ground-state cooling when combined with cryogenics [37].

\section{Acknowledgements}

We thank Jack Harris for sharing his many insights about optomechanical systems. F. M. also thanks B. Kubala, M. Ludwig and C. Neuenhahn for discussions on this topic. F. M. thanks the DFG for support via SFB 631, the EmmyNoether program, and the NIM cluster of excellence. The work of S. M. G. was supported by the NSF via grants DMR-0603369 and DMR-0653377. A. A. C. would like to thank the Canadian Institute for Advanced Research (CIFAR).

\section{References}

[1] V.B. Braginsky and A.B. Manukin. Ponderomotive effects of electromagnetic radiation. Soviet Physics JETP, 25:653, 1967.

[2] V. B. Braginsky, A. B. Manukin, and M. Yu. Tikhonov. Investigation of dissipative ponderomotive effects of electromagnetic radiation. Soviet Physics JETP, 31:829, 1970.

[3] A. Dorsel, J. D. McCullen, P. Meystre, E. Vignes, and H. Walther. Optical bistability and mirror confinement induced by radiation pressure. Phys. Rev. Lett., 51:1550, 1983.

[4] P. F. Cohadon, A. Heidmann, and M. Pinard. Cooling of a mirror by radiation pressure. Phys. Rev. Lett., 83:3174, 1999.

[5] D. Kleckner and D. Bouwmeester. Sub-kelvin optical cooling of a micromechanical resonator. Nature, 444:75, 2006. 
[6] M. Vogel, C. Mooser, K. Karrai, and R. J. Warburton. Optically tunable mechanics of microlevers. Applied Physics Letters, 83:1337, 2003.

[7] C. Höhberger-Metzger and K. Karrai. Cavity cooling of a microlever. Nature, 432:1002, 2004.

[8] O. Arcizet, P. F. Cohadon, T. Briant, M. Pinard, and A. Heidmann. Radiation-pressure cooling and optomechanical instability of a micromirror. Nature, 444:71, 2006.

[9] S. Gigan et al. Self-cooling of a micromirror by radiation pressure. Nature, 444:67, 2006.

[10] A. Schliesser, P. Del'Haye, N. Nooshi, K. J. Vahala, and T. J. Kippenberg. Cooling of a micro-mechanical oscillator using radiation pressure induced dynamical back-action. Phys. Rev. Lett., 97:243905, 2006.

[11] Thomas Corbitt et al. Toward achieving the quantum ground state of a gram-scale mirror oscillator. Phys. Rev. Lett., 98:150802, 2007.

[12] J. D. Thompson, B. M. Zwickl, A. M. Jayich, F. Marquardt, S. M. Girvin, and J. G. E. Harris. Strong dispersive coupling of a high finesse cavity to a michromechanical membrane. Nature, 452:72, 2008.

[13] F. Marquardt, J. G. E. Harris, and S. M. Girvin. Dynamical multistability induced by radiation pressure in high-finesse micromechanical optical cavities. Phys. Rev. Lett., 96:103901, 2006.

[14] T. Carmon, H. Rokhsari, L. Yang, T. J. Kippenberg, and K. J. Vahala. Temporal behavior of radiation-pressure-induced vibrations of an optical microcavity phonon mode. Phys. Rev. Lett., 94:223902, 2005.

[15] T. J. Kippenberg, H. Rokhsari, T. Carmon, A. Scherer, and K. J. Vahala. Analysis of radiation-pressure induced mechanical oscillation of an optical microcavity. Phys. Rev. Lett., 95:033901, 2005.

[16] C. Höhberger and K. Karrai. Self-oscillation of micromechanical resonators. Nanotechnology 2004, Proceedings of the 4th IEEE conference on nanotechnology, page 419, 2004.

[17] Max Ludwig, Clemens Neuenhahn, Constanze Metzger, Alexander Ortlieb, Ivan Favero, Khaled Karrai, and Florian Marquardt. Self-induced oscillations in an optomechanical system. arXiv:0711.2661, 2007.

[18] T. J. Kippenberg and K. J. Vahala. Cavity opto-mechanics. Optics Express, 15:17172, 2007.

[19] K. R. Brown, J. Britton, R. J. Epstein, J. Chiaverini, D. Leibfried, and D. J. Wineland. Passive cooling of a micromechanical oscillator with a resonant electric circuit. Phys. Rev. Lett., 99:137205, 2007. 
[20] A. A. Clerk and S. Bennett. Quantum nanoelectromechanics with electrons, quasi-particles and Cooper pairs: effective bath descriptions and strong feedback effects. New Journal of Physics, 7:238, 2005.

[21] M. P. Blencowe, J. Imbers, and A. D. Armour. Dynamics of a nanomechanical resonator coupled to a superconducting single-electron transistor. New Journal of Physics, 7:236, 2005.

[22] A. Naik et al. Cooling a nanomechanical resonator with quantum backaction. Nature, 443:193, 2006.

[23] S. D. Bennett and A. A. Clerk. Laser-like instabilities in quantum nanoelectromechanical systems. Phys. Rev. B, 74:201301, 2006.

[24] D. A. Rodrigues, J. Imbers, and A. D. Armour. Quantum dynamics of a resonator driven by a superconducting single-electron transistor: a solidstate analogue of the micromaser. Phys. Rev. Lett., 98:067204, 2007.

[25] D. A. Rodrigues, J. Imbers, T. J. Harvey, and A. D. Armour. Dynamical instabilities of a resonator driven by a superconducting single-electron transistor. New Journal of Physics, 9:84, 2007.

[26] C. A. Regal, J. D. Teufel, and K. W. Lehnert. Measuring nanomechanical motion with a microwave cavity interferometer. arXiv:0801.1827, 2008.

[27] D. Meiser and P. Meystre. Coupled dynamics of atoms and radiationpressure-driven interferometers. Phys. Rev. A, 73:033417, 2006.

[28] K. W. Murch, K. L. Moore, S. Gupta, and D. M. Stamper-Kurn. Measurement of Intracavity Quantum Fluctuations of Light Using an Atomic Fluctuation Bolometer. arXiv:0706.1005v2, 2007.

[29] S. Bose, K. Jacobs, and P. L. Knight. Scheme to probe the decoherence of a macroscopic object. Phys. Rev. A, 59:3204, 1999.

[30] William Marshall, Christoph Simon, Roger Penrose, and Dik Bouwmeester. Towards quantum superpositions of a mirror. Physical Review Letters, 91(13):130401, 2003.

[31] M. Pinard, A. Dantan, D. Vitali, O. Arcizet, T. Briant, and A. Heidmann. Entangling movable mirrors in a double-cavity system. Europhysics Letters, 72:747, 2005.

[32] F. Marquardt, J. P. Chen, A. A. Clerk, and S. M. Girvin. Quantum theory of cavity-assisted sideband cooling of mechanical motion. Phys. Rev. Lett., 99:093902, 2007.

[33] I. Wilson-Rae, N. Nooshi, W. Zwerger, and T. J. Kippenberg. Theory of ground state cooling of a mechanical oscillator using dynamical back-action. Phys. Rev. Lett., 99:093901, 2007. 
[34] I. Favero and K. Karrai. Cavity cooling of a nanomechanical resonator by light scattering. arXiv:0707.3117, 2007.

[35] K. Karrai, I. Favero, and C. Metzger. Doppler controlled dynamics of a mirror attached to a spring. arXiv:0706.2841, 2007.

[36] A. Schliesser, R. Riviere, G. Anetsberger, O. Arcizet, and T. J. Kippenberg. Resolved sideband cooling of a micromechanical oscillator. arXiv:0709.4036, 2007.

[37] S. Gröblacher, S. Gigan, H. R. Böhm, A. Zeilinger, and M. Aspelmeyer. Radiation-pressure self-cooling of a micromirror in a cryogenic environment. Europhys. Lett., 81:54003, 2008. 